\title{
Initial particle velocity spatial distribution from 2-D erupting bubbles in fluidized beds
}

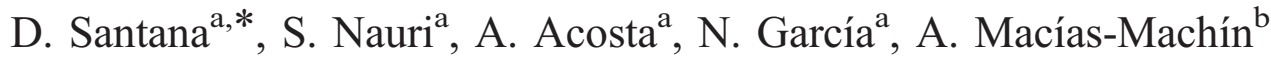 \\ ${ }^{a}$ Universidad Carlos III de Madrid, Area de Ingeniería Térmica, Avda. de la Universidad 30, 28911 Leganes, Madrid, Spain \\ ${ }^{\mathrm{b}}$ Universidad de Las Palmas de Gran Canaria, Campus de Tafira Alta, s/n Las Palmas de Gran Canaria, Spain
}

\begin{abstract}
Some results on particle image velocimetry (PIV) in 2 D freely bubbling fluidized beds are presented. The PIV applications were used in order to determine the initial particle velocity of bubble eruptions. A two dimensional non reacting fluidized bed was constructed to measure the origin of the ejected particles and the initial particle velocity distribution, using coarse sand particles. The bubble ejection mechanism was observed taking into account the origin of particles ejected, the initial particle velocity distributions as well as the effect of other neighbor exploding bubbles. Our results show that the assumption of linear dependence of initial velocity with the angle predicts the velocity faithfully only for purely vertical ascent bubbles. Measurements of ejection velocities show that initial velocities in the combined layer are higher than those of the particles in the nose of the leading bubble. Avoiding coalescence of bubbles at the bed surface can lead to less particle entrainment out of the bed and consequently to shorter fluidized beds.
\end{abstract}

Keywords: Bubbling fluidized beds; Particle ejection velocity; Particle image velocimetry (PIV)

\section{Introduction}

Among the several techniques for gas-solid reaction available, fluidized beds yield the greatest rate of reaction per reactor volume. This is mainly due to the high contact area between particles and gas. Thus, it is not surprising to find a wide range of applications of this technique such as combustion, gasification, incineration, cracking catalysis, etc.

The flow in a fluidized bed is very complex because of its two-phase non-steady 3D characteristics. Hence, experimental measurements and numerical models are far from complete.

One of the most pressing problems in the study of fluidized beds is the entrainment and/or elutriation of the particles. Entrainment and elutriation are technical terms used interchangeably to describe the loss of inert particles and/or fuel in a fluidized bed system. Normally, entrainment

\footnotetext{
* Corresponding author. Tel.: +34 916249158.

E-mail address: dsantana@ing.uc3m.es (D. Santana).
}

is the carryover of large ejected particles by the gas flow field. Eventually, the particles fall back to the bed surface because of their high terminal settling velocities, elutriation is normally used to describe the fractional carryover of finer particles when the bed particles size distribution is wide.

Particle elutriation and entrainment are extremely complex phenomena, which are not well understood from a dynamic point of view [1]. Most of the available information consists of correlations of experimental data giving the elutriation rate as a function of the physical characteristics of gas and particles, besides operating conditions. To properly model the entrainment flow, information about two basic processes is needed: the dynamics of the particle when ejected from bubble eruption to the open surface of the bed and its path through the freeboard.

Entrainment flow simulations for freely gas-solid fluidized beds are based on particle trajectories in the freeboard. Thus, the initial particle velocity distributions of the bubble eruption at the bed surface must be known. An accurate prediction of initial particle velocities and gas velocity field can lead to a more precise determination of particle 
concentrations along the freeboard and consequently, to a reliable design of filtering equipment such as cyclones, scrubbers and bag filters.

The initial particle velocity spatial distribution at the bed open surface is crucial for the accurate prediction of the particle trajectory in the freeboard and to determine the maximum height attained by the ejected particles. It is believed that the initial particle velocity direction has a distribution with respect to the vertical direction. Peters and Prybylowski [2] assumed that the initial particle velocity magnitude distribution is constant and its direction is normal to the bubble surface, right at the moment of the bubble bursting. Demmich [3] suggested that the particle velocity magnitude distribution along the circumference of the bubble nose should be a decaying exponential function. However, no concrete velocity distribution was given. On the other hand, Fung and Hamdullahpur [4] assumed that it decreases linearly with the bubble angle, $\theta$, when measured from the vertical direction, see Fig. 5. None of these authors presents experimental results about initial particle velocity, neither do they give a clear definition of when the initial value of the particle velocity can be defined.

Most of the available results about the initial particle velocity distribution are related to the origin of ejected particles and the bubble eruption mechanism [5]. These results are obtained from measurements during the eruptions of bubbles injected in narrow fluidized beds with coarse particles. The size of the bubbles produced by a given gas injection is very sensitive to both overall flow rate and degree of packing or disturbance in the dense phase, at least with some materials. Therefore, it is difficult to translate data related to the initial particle velocities obtained from carefully injected bubbles to the ones obtained from ejected particles in freely bubbling fluidized beds. These results about the origin of ejected particles and bubble dynamics in fluidized beds have been obtained mostly in 2D fluidized beds, as 3D fluidized beds visual observations of bubble dynamics are impossible today, except for special experimental techniques as X-ray image [6] and $\gamma$-radiation [7].

Regarding the origin of particles elutriated, there exits a controversy as to which part of the bubbles, bubble dome or its wake, causes ejection. George and Grace [8] segregated a thick layer of coke at the surface of a fluidized bed filled with sand, injected a single bubble into the bed and captured the ejected particles at several positions above the bed surface. They found that few coke particles were captured and concluded that ejected particles came from bubble wake. Later, Pemberton and Davidson [9] proposed an alternative conclusion about this result. They suggested that the few particles captured were due to drain-off of coke particles from the bubble dome before it burst. Saxena and Mathur [10] confirmed this last conclusion. They identified the bulge-burst or nose-burst phenomenon as characteristic of single-bubble burst.
Early measurements in fluidized beds were restricted to the freeboard region using point measurements techniques, such as X-ray photography [6], cine films [11], laser Doppler anemometry (LDA) [12] and hot wire anemometry [9]. These yields a time history in a point of the bed, normally far from the bed surface, from which mean and RMS-fluctuation velocities can be calculated. A disadvantage is that the readings at different points in the flow are not simultaneous. This is not a problem if the flow being measured is reproducible. But, above the fluidized bed, the velocity fluctuations appear to be large and chaotic. Therefore measurements of gas and/or particles velocities at different positions in the freeboard and/or the bed surface, taken at different times, may not be fully comparable. In these conditions, in fact, the flow fields might not be identical.

As a consequence, particle image velocimetry (PIV) techniques have been progressively used to study fluidization phenomena. This technique yields a simultaneous particle average displacement field covering a wide region of the flow. The first application of PIV to fluidization was made by Rix et al. [13]. The experiments were restricted to the determination of the gas field in the freeboard above an erupting bubble but far from the bed surface. YórquezRamírez and Duursma [14,15] and Duursma et al. [16] studied the air flow pattern above an erupting bubble in an incipiently fluidized bed, showing the applicability of PIV techniques to obtain the continuous phase flow fields in fluidized beds.

A two-dimensional non-reacting fluidized bed was constructed with the aim of measuring the origin of ejected particles and the initial particle velocity distribution in a freely fluidized bed. The bubble ejection mechanism was observed taking into account the origin of ejected particles, the initial particle velocity distributions as well as the effect of other neighbor exploding bubbles. Images for different bubble eruption mechanism were selected, both isolated and affected by other neighbor exploding bubbles.

PIV measurements of the particles sliding on the surface of the glass sheet end walls are reported in this paper. Questions arise about the validity of the velocity fields obtained, as representative of the velocity of the particles inside the bed. Solid friction could impede these particles. In the experiments performed, this does not seem the case, as the interface of the bubbles seems neatly perpendicular to the end walls. Besides, end wall friction could not be great enough to separate contacting particles, as a consequence of a higher entrainment of the free neighbor particles, without changes in the whole aggregate shape. This point is still unclear and under investigation.

This paper presents the results obtained with an advanced PIV technique applied to initial particle velocity determination on bubble eruptions in a 2-D freely bubbling fluidized bed. This advanced PIV technique allows successful measurements to be obtained in the very challenging fluidized bed environment. 


\section{Experimental}

In Fig. 1, the experimental setup can be observed. A twodimensional open top fluidized bed of dimensions $100 \times 100 \times 0.5 \mathrm{~cm}$ was constructed from glass sheet. The solids in the bed were fluidized by compressed air supplied from the mains. A plate with 100 orifices $1 \mathrm{~mm}$ diameter served as a distributor. A plenum $30 \mathrm{~cm}$ height with a predistributor was mounted to ensure a uniform air velocity distribution at the entrance of the plate distributor.

Quartz natural sand particles with mean diameter $300 \mu \mathrm{m}$ was used as fluidized medium. The settled bed depth was 14 $\mathrm{cm}$. Minimum fluidizing velocity was $57.3 \mathrm{~cm} / \mathrm{s}$. All the experiments presented here were carried out at $160 \mathrm{~cm} / \mathrm{s}$ in the free bubbling regime. In this way, since the particle terminal settling velocities were higher than the superficial gas velocity, particle entrainment out of the fluidized bed was avoided.

The bed was uniformly illuminated by a continuous lateral light source. The bubble approach to the surface and the bubble eruptions images were captured using a Kodak Motion Corder Analyzed SR series CCD video camera at a rate of $250 \mathrm{~Hz}$. The imaged area was $15 \times 16$ $\mathrm{cm}(480 \times 512$ pixels $)$.

The video camera and the light source were positioned on the same side of the bed. To avoid noise and external interferences within the bubble path measurements, a black sheet was placed over the back side of the bed. This leads to images with white particles on a black background.

Series of recorded bubble eruptions were analyzed via correlations based on the LFC-PIV technique [17]. This is an iterative image deformation method that progressively reduces the average particle displacement between two consecutive images increasing, in this way, the signal to noise ratio. With this method, the spatial wavelength resolution can be reduced down to the average distance between particles. The interrogation window is suitably weighted in order to avoid instabilities in the displacement reduction process. For the LFC-PIV implementation, an 8pixel grid distance between measurements was selected. This makes the non-weighted effective window side size 8 pixels. The number of iterations of the LFC-PIV system was limited from 3 to 5, depending on the case. This allows for the description of 15 pixels size features [18].

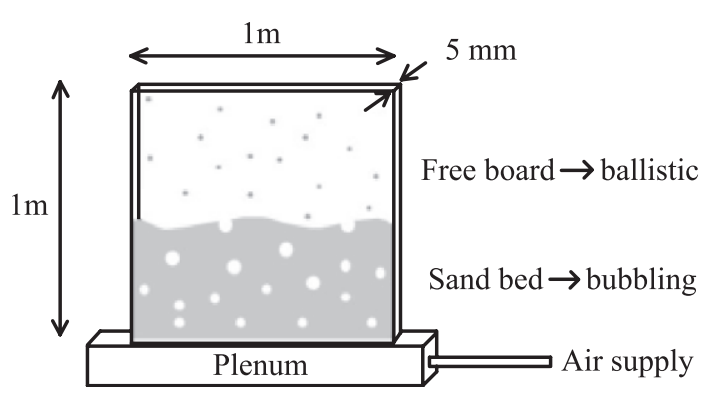

Fig. 1. Experimental setup.

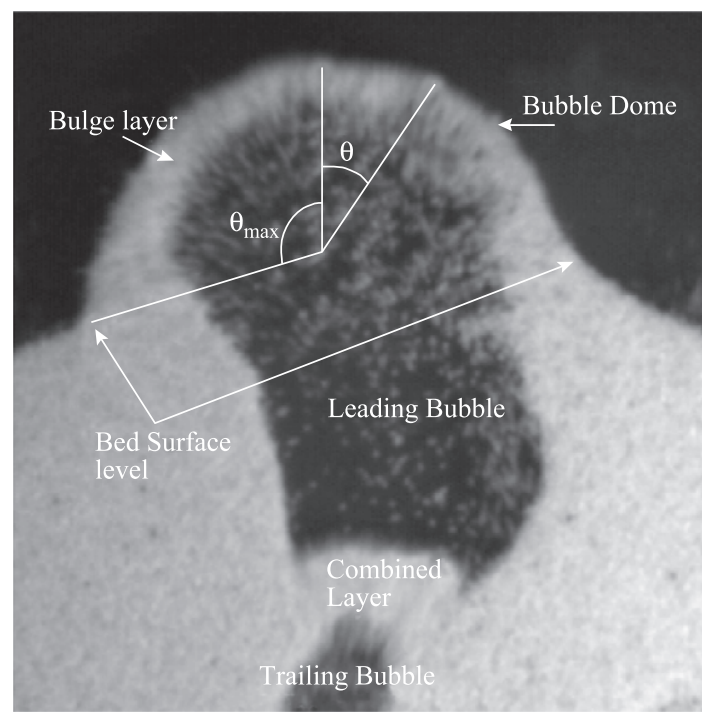

Fig. 2. Regions of interest during the bubble bursting.

To evaluate the evolution of the bulge layer, a global gray threshold was applied to the images. Only vectors in the bulge layer and in the combined layer are presented. This can be accomplished readily as the emulsion phase possesses higher gray-level intensity values in comparison to the bubble phase. In Fig. 2 are defined the regions of interest during the bubble bursting.

\section{Results and discussion}

\subsection{Mechanism of solids ejection}

It is normal to use the size of the bubble in order to explain the mechanism of solids ejection. Our measurements show that it is also important to consider the shape of the bubble as well as the direction of the ejection bulk motion. This can be seen in Fig. 3 where the velocity fields of a round shaped bubble and of a stretched one are represented. The maximum velocity reached during the ejection appears to be higher in the second case and its distribution seems to depend on the ejection direction.

The images reported in Fig. 4 (4 ms elapsed time) evidence that it is also important to consider the effect of other neighbouring exploding bubbles in the study of the ejection mechanism. The asymmetry of the velocity distribution seems to be, in this case, due to the interaction between the vertically ejected bubble and the neighboring ones inside the fluidized bed and the falling particle of the previously erupted ones.

For the operating conditions used in the experiments, bubble coalescence at the bed surface was shown to be the most important solids ejection mechanism. When the bubbles coalesce at the bed surface, a series of events occurs. The bulge layer of the leading bubble stretches and moves upward as the bubble approaches the bed surface, 
(a)

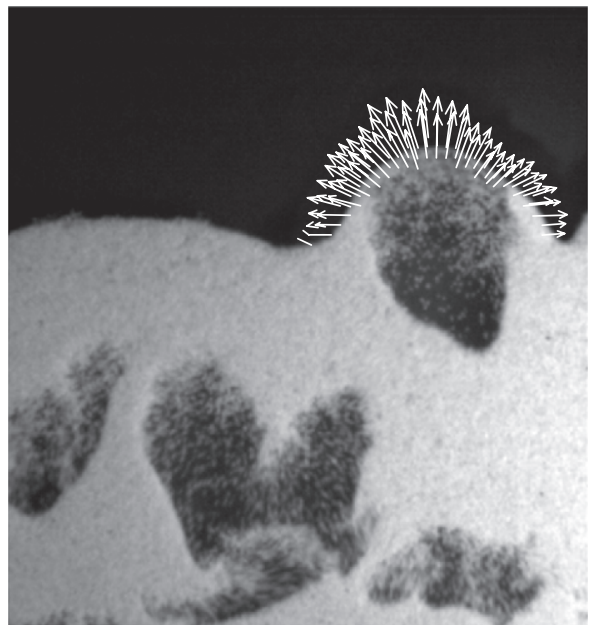

(b)

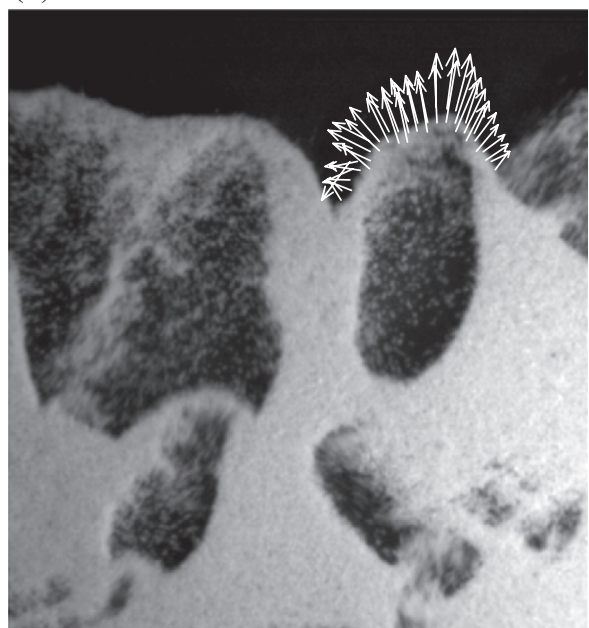

Fig. 3. Velocity field for a round (a) and a stretched (b) bubble.

becoming leaner. After rising to a maximum height somewhat less than the bubble diameter, the bubble particles fall back to the bed surface, eventually toward the bubble interior. The particles of the leading bubble are thrown above the bed as a result of the nose breakup [19]. The layer of solids between the bubbles combines with the leading (a)

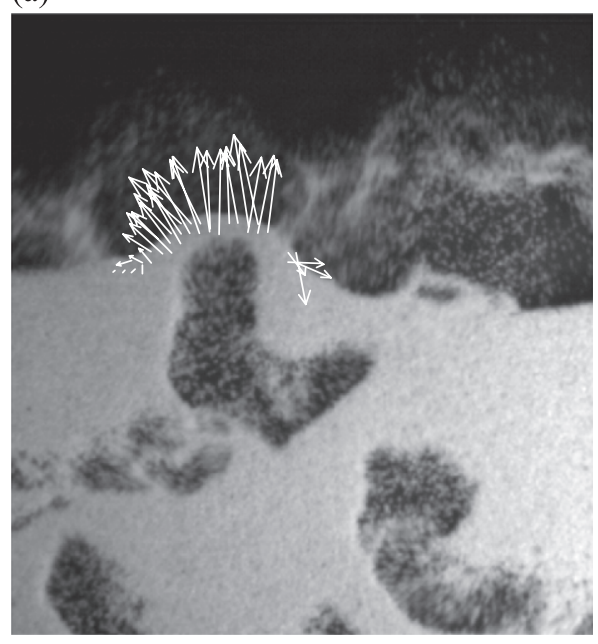

(c)

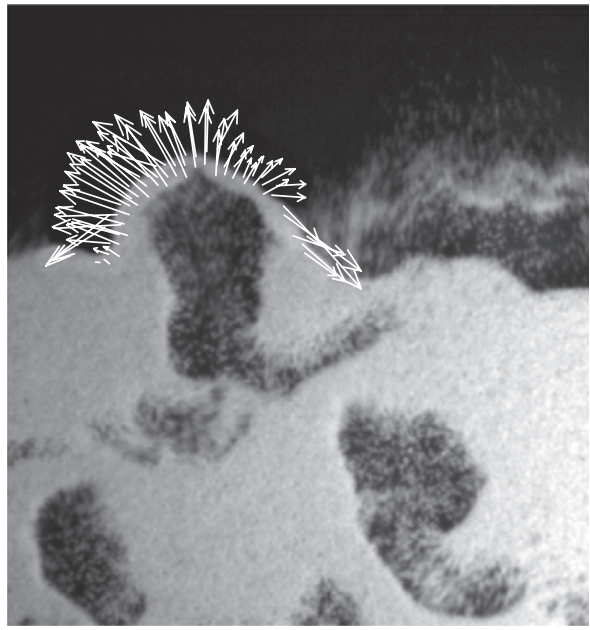

(b)

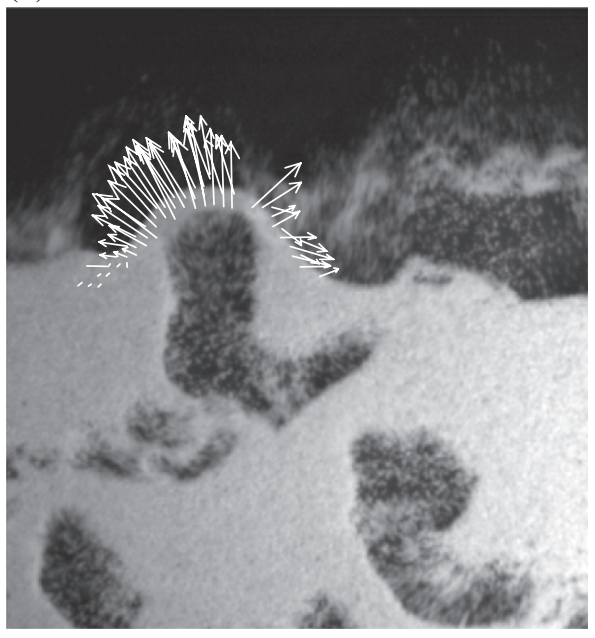

(d)

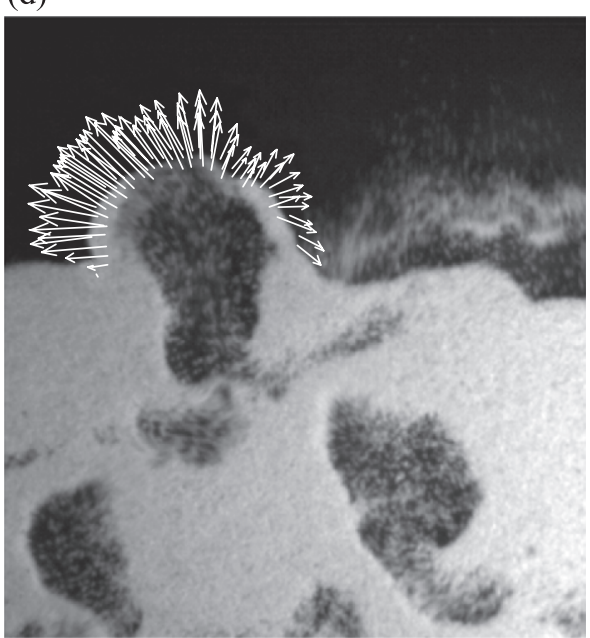

Fig. 4. Influence of the neighboring bubbles on the ejecting mechanism and the velocity field. 
bubble bulge layer. The resulting particle cloud expands upward and outward through the channel opened by the leading bubble and falls back to the bed surface. Fig. 5 depicts this phenomenon.

Fig. 5 can explain the alternative conclusion of Pemberton and Davidson [9] to describe George and Grace experimental [8] results. As the bubble approaches to the bed surface a dome is formed, particles are drained from the dome and consequently particles on the bulge are ejected as the bubble bursts [20]. When the bubble approaches to the bed surface particles are not only drained from the dome but they are also displaced from the bubble nose. Moreover, particles located on the top of the bed surface at the instant of dome formation have less probability to be ejected vigorously than these in the bulge.

Fig. 5(b) shows that the ejected particles are originated at the nose of bursting bubble and/or at the combined layer, and move quite independently [11], at least in the range of operating conditions described here.

\subsection{Initial particle velocity for the leading bubble}

Fig. 6 shows the evolution of the bubble nose velocities while the bulge layer of the bubble stretches and expands at the bed surface. For clearness, only the vector evolution at the bubble nose is represented.

As the bubble approaches the bed surface, the particles at the top of the nose accelerate upward while the ones at the bottom decelerate, falling off towards the inside of the bubble. Through this mechanism the bubble nose layer becomes thinner and thus lighter. The initial particle velocity can be defined as the velocity at which particles begin to behave individually rather than particle aggregates.

Fig. 6 shows how the bubble dome area increases above the bed surface. The bubble dome accelerates in all directions except in the region near the bed surface level, where bubble dome intersect the emulsion phase. The acceleration is small until the bubble mass center is at the bed surface level. The bubble nose accelerates until its layer (a)

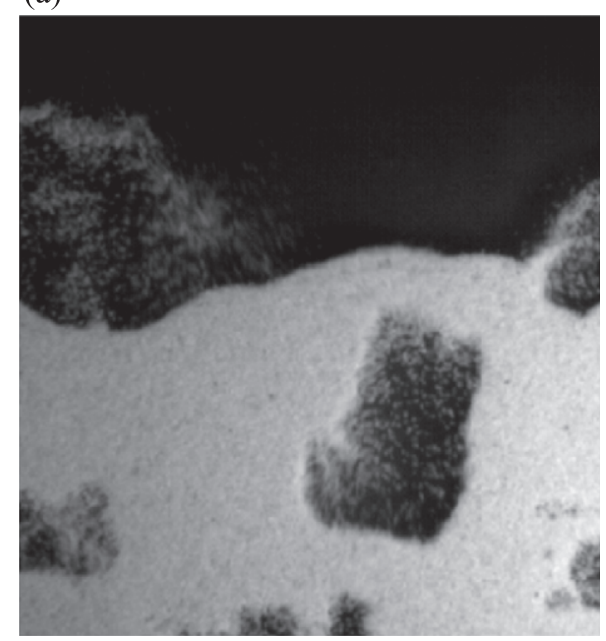

(c)

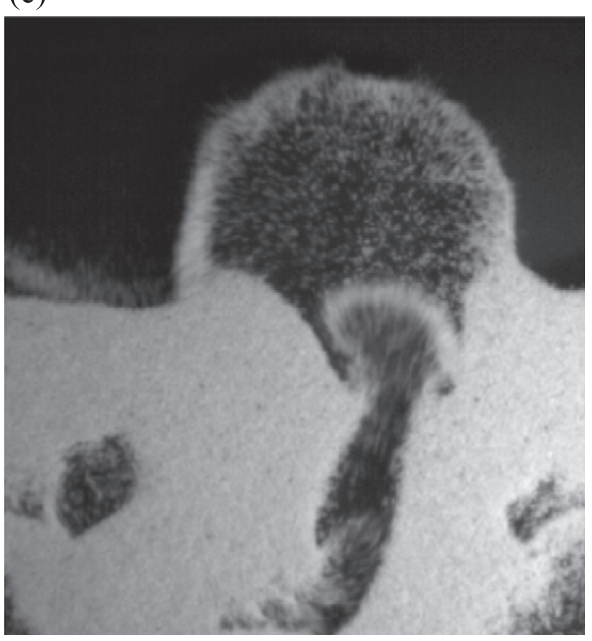

(b)

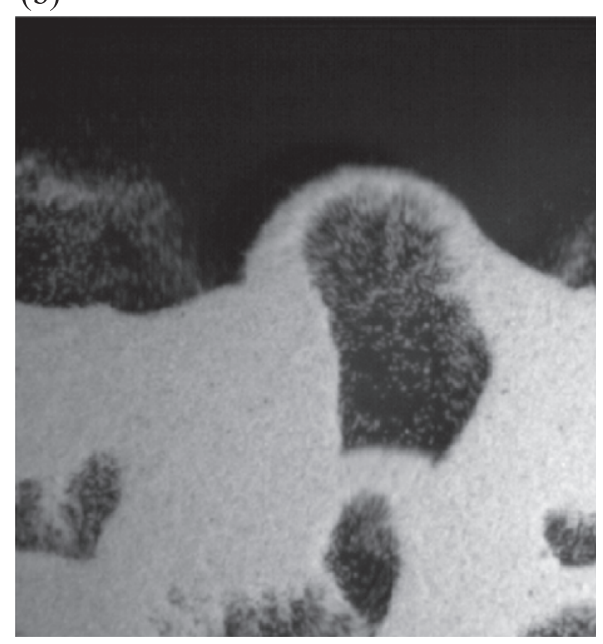

(d)

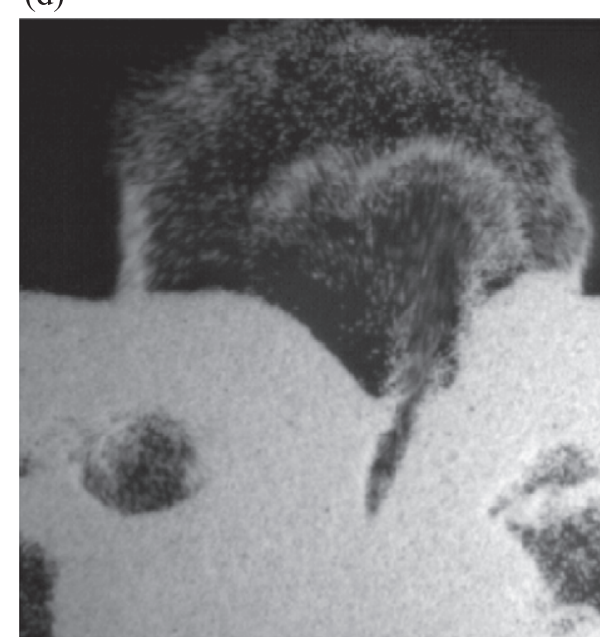

Fig. 5. Mechanism of solids ejection showing the particles in white: (a) leading bubble approaching the bed surface, (b) bubble erupting at bed surface, (c) combined layer of solids growing in size and (d) combined layer of solids ejection. 


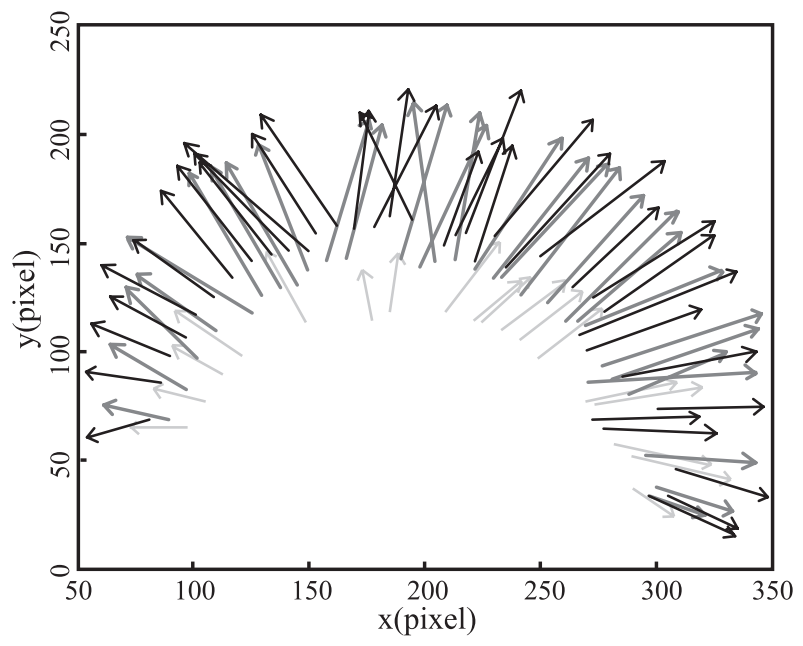

Fig. 6. Velocity evolution at consecutive times of the leading bubble layer.

becomes more porous and the particles are dragged to the bed freeboard. According to Do et al. [11], the ejection velocity of the particles was obtained from the rate of rise of the particles on the top of the advancing bubble as the bubble breaks the surface. The corresponding velocity is what we define as initial particle ejection velocity (vectors in black in Fig. 6).

There are no measurements available in the literature of the spatial distributions of initial particle ejection velocity. Nevertheless various authors have proposed distributions from a theoretical point of view. Taking into account the porosity variation along the bulge, Peters and Prybylowski [2] assumed a uniform initial ejection velocity distribution along the bubble dome. Demmich [3] supposed an exponential distribution based on the force balance at the bubble nose layer. Fung and Hamdullahpur [4] proposed that the radial particle ejection velocity on the bubble circumference is such that ejection velocity decreases linearly with the angle $\theta$. According to the last mentioned authors, the initial particle distribution can be estimated as a sine-cosine function:

$\frac{U}{U_{\max }}=\left(1-\frac{\theta}{\theta_{\max }}\right) \cos (\theta)$

$\frac{U}{U_{\max }}=\left(1-\frac{\theta}{\theta_{\max }}\right) \sin (\theta)$

Where $\theta_{\max }$ is the maximum particle ejection angle, $U$ and $V$ are the vertical and horizontal velocity vector components, respectively, and $U_{\max }$ is the maximum vertical velocity along the bubble nose.

Among these initial particle ejection velocity distributions, the linear assumption seems more appropriate than the others to represent the ejection velocities resulting from the PIV measurements performed.

In Figs. 7 and 8, each experimental ejection velocity component obtained by PIV are represented together with Fung and Hamdulampur Eqs. (1) and (2). As shown in Fig.

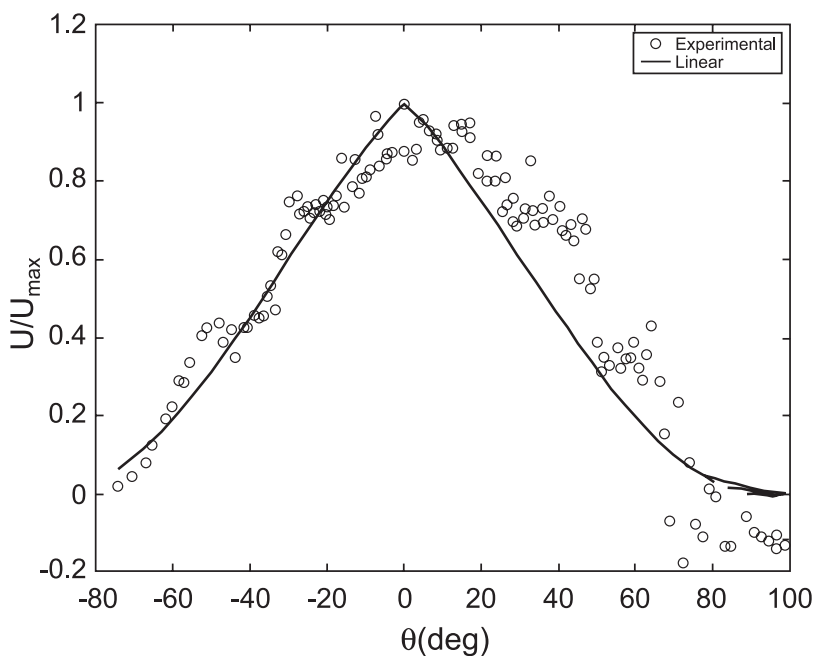

Fig. 7. Vertical ejection particle velocity.

7, the Fung and Hamdullahpur [4] distribution describes faithfully the ejection velocities resulting from the PIV measurements, in the case of a bubble erupting vertically. The deviation between the experimental results and the theoretical assumption (Fig. 7) is due to the bubble shape and to its approach to the bed surface (Fig. 2).

Regarding the horizontal component of the ejection velocity, the linear assumption does not seem so appropriate (Fig. 8). The experimental results show also that the velocity at the bubble nose top is purely vertical, because the horizontal component magnitude is negligible. Its value is comparable with the horizontal one at the level of the supporting layer (bed). Discrepancies between the horizontal component magnitude were owing to the fact that bed surface was not perfectly horizontal at the moment of particle ejections.

The PIV measurements show that this is only valid for bubbles approaching vertically to the bed surface. In order to predict the velocity measurements for bubbles approach-

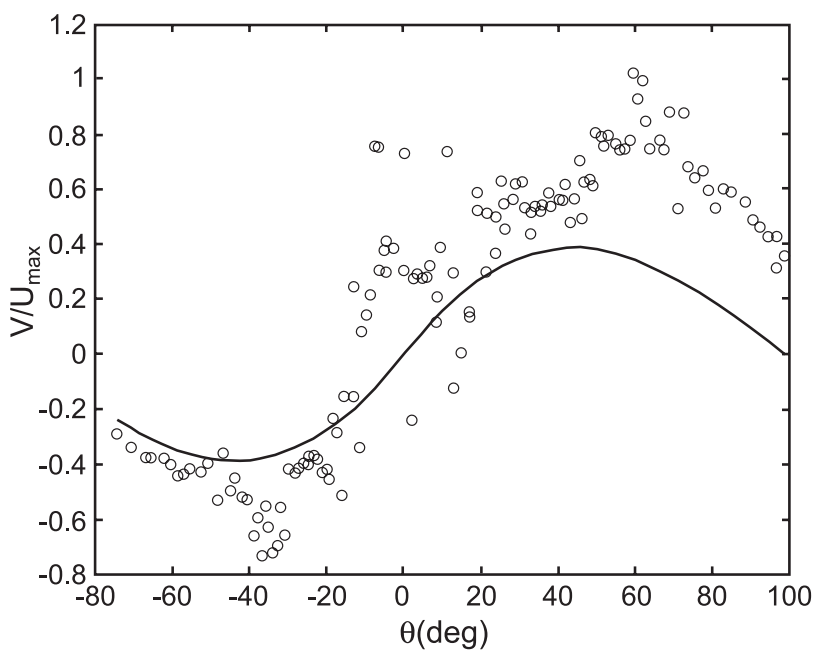

Fig. 8. Horizontal ejection particle velocity. 
ing in a different direction (Fig. 3), no theoretical model has been developed.

\subsection{Particle velocities for the trailing bubble}

The velocity evolution of the combined layer between the leading and trailing bubbles is shown in Fig. 9. The wake of the leading bubble acts as a bed surface for the trailing bubble nose (a). In proportion, as the trailing bubble approach the leading bubble, the combined layer accelerates through the channel opened by the leading bubble (b), reaching a maximum velocity when the combined layer reaches the divergent channel opened by the leading bubble (c); progressively, the combined layer decelerates, catching the particles ejected by the leading bubble (d).

As the sequence shown in Fig. 9, it can be observed how the bubble eruption caused by coalescence at the bed surface, has a significant effect on the ejection of solids particles [21]. When two bubbles coalesce right at the bed surface, particles in the combined layer are ejected with a initial velocities considerably greater than those caused by single bursting bubbles [11]. These initial velocities are at least twice the ejection velocities in the nose of the leading bubble.

\section{Conclusions}

It has been possible to measure individual velocities of assembled particles using advanced correlation PIV in an opaque two-phase flow of a 2-D bubbling fluidized bed.

The linear dependence with the angle of the velocity magnitude predicts well the initial ejection velocities for a bubble erupting vertically and also for low angles with the verticality. For higher angles, the particles move parallel to the bed surface with a negligible vertical velocity vector.

Particles in the combined layer possess ejection velocities much higher than those of particles ejected from the leading bubble nose.

The use of the most advanced PIV techniques opens new possibilities in assessing the dynamics of complex twophase flows. (a)

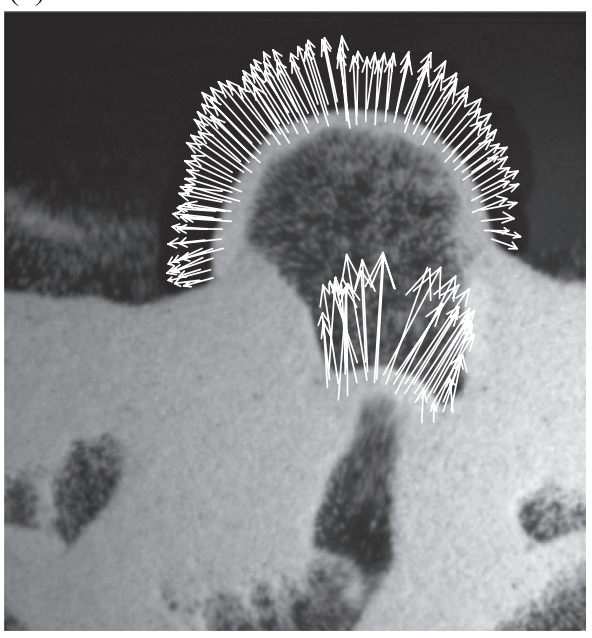

(c)

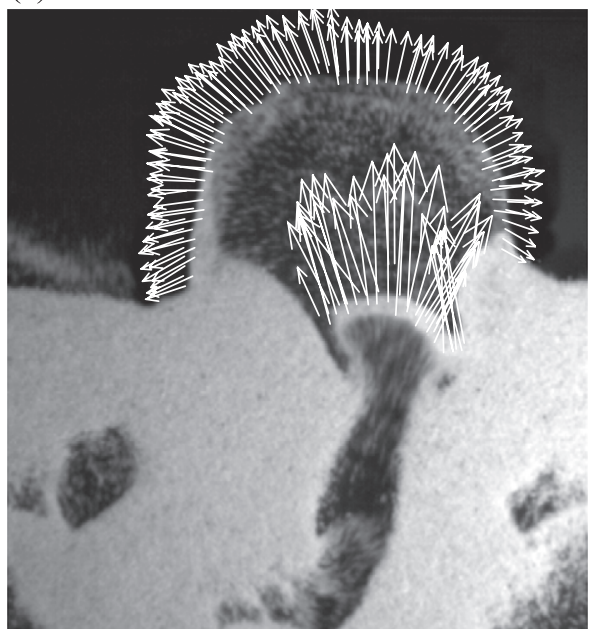

(b)

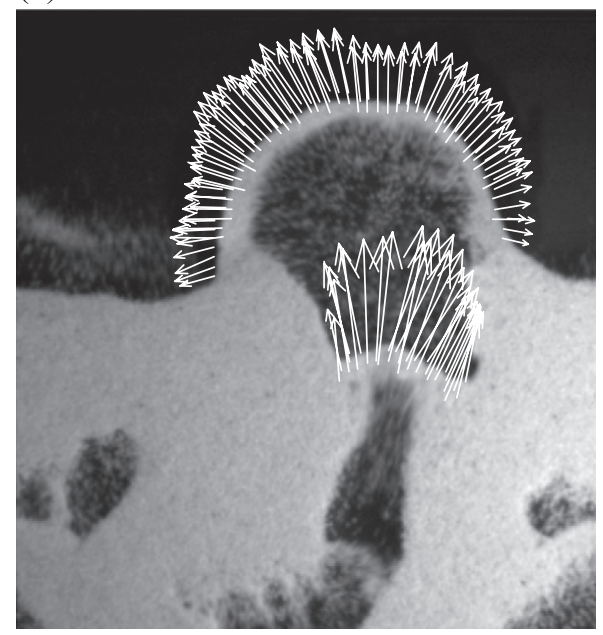

(d)

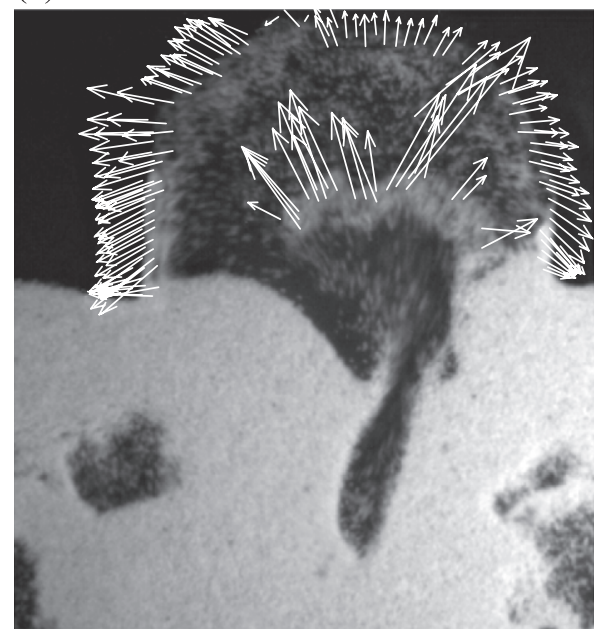

Fig. 9. Velocity field of the combined layer at consecutive time steps. 


\section{Acknowledgements}

The financial support from Ministerio de Ciencia y Tecnología of the Spanish Government is appreciated (project number DPI2002-02453).

\section{References}

[1] R. Jan de Korte, J.C. Schouten, C.M. Van den Bleek, Controlling bubble coalescence in a fluidized-bed model using bubble injection, AIChE J. 47 (2001) 851860.

[2] M.H. Peters, D.L. Prybylowski, Particle motion above the surface of a fluidized bed: multiparticle effects, 79 (1983) 8386 .

[3] J. Demmich, Mechanism of solids entrainment from fluidized beds, Ger. Chem. Eng. 7 (1984) 386394.

[4] A.S. Fung, F. Hamdullahpur, A gas and particle flow model in the freeboard of a fluidized bed based on bubble coalescence, Powder Technol. 74 (1993) 121133

[5] E.K. Levy, H.S. Caram, J.C. Dille, S. Edelstein, Mechanism for solids ejection from gas-fluidized beds, AIChE J. 29 (1983) 383388

[6] P.N. Rowe, B.A. Partridge, An X-ray study of bubbles in fluidised beds, Trans. Inst. Chem. Eng. 43 (1965) T157 T175.

[7] A.W. Weimer, D.C. Gyure, D.E. Clough, Application of a gammaradiation density gauge for determining hydrodynamic properties of fluidized beds, Powder Technol. 44 (1985) 179194.

[8] S.E. George, J.R. Grace, Entrainment of particles from aggregative fluidized beds, AIChE Symp. Ser. 74 (1978) 6774.

[9] S.T. Pemberton, J.F. Davidson, Turbulence in the freeboard of a gasfluidised bed, Chem. Eng. Sci. 39 (1984) 829840.

[10] S.C. Saxena, A. Mathur, On the origin of solids projected from the surface of a gas-fluidized bed, Chem. Eng. Sci. 44 (1984) 917918.
[11] H.T. Do, J.R. Grace, R. Clift, Particle ejection and entrainment from fluidised beds, Powder Technol. 6 (1972) 195200

[12] Y. Levy, F.C. Lockwood, Laser Doppler measurements of flow in freeboard of a fluidized bed, AIChE J. 29 (1983) 889894.

[13] S.L.J. Rix, D.H. Glass, C.A. Greated, Preliminary studies of elutriation from gas-fluidised beds using particle image velocimetry, Chem. Eng. Sci. 51 (1996) 34793489.

[14] M.I. Yórquez-Ramírez, G.R. Duursma, Study of the flow pattern above an erupting bubble in a incipiently fluidised bed using image shifting, Chem. Eng. Sci. 55 (2000) 20552064.

[15] M.I. Yórquez-Ramírez, G.R. Duursma, Insights into the instantaneous freeboard flow above a bubbling fluidised bed, Powder Technol. 116 (2001) 7684

[16] G.R. Duursma, D.H. Glass, M.I. Yórquez-Ramírez, PIV investigations of flow structures in the fluidised bed freeboard region, Powder Technol. 120 (2001) 2 11.

[17] J. Nogueira, A. Lecuona, P.A. Rodríguez, Local field correction PIV, implemented by means of simple algorithms, and multigrid versions, Meas. Sci. Technol. 12 (2001) 19111921.

[18] A. Lecuona, J. Nogueira, P.A. Rodriguez, D. Santana, Accuracy and time performance of different schemes of the Local Field Correction (LFC) PIV technique, Exp. Fluids 33 (2002) 743751

[19] S.C. Saxena, A. Mathur, A model for the projection of solid particles from the surface of a gas-fluidized bed, Chem. Eng. Sci. 39 (1989) 774777.

[20] S.T. Pemberton, J.F. Davidson, Elutriation from fluidized beds: I. Particle ejection from the dense phase into the freeboard, Chem. Eng. Sci. 41 (1986) 243251

[21] H. Hatano, T. Okuma, M. Ishida, Bubble eruption model for entrainment phenomena below the THD for gas solid fluidized beds, J. Chem. Eng. Jpn. 17 (1984) 491497. 